Please do not remove this page

RMIT

UNIVERSITY

\title{
Integration of microfluidic channels and optical waveguides using low-cost polymer microfabrication techniques
}

Mitchell, Arnan; Holland, Anthony; Vora, Kaushal; Ghantasala, Muralidhar

https://researchrepository.rmit.edu.au/esploro/outputs/9921857843401341/filesAndLinks?institution=61RMIT_INST\&index=null

Mitchell, A., Holland, A., Vora, K., \& Ghantasala, M. (2004). Integration of microfluidic channels and optical waveguides using low-cost polymer microfabrication techniques. Proceedings of the IEEE Biophotonics Summer Topical Conference 2004. https://doi.org/10.1109/LEOSST.2004.1338655

Published Version: https://doi.org/10.1109/LEOSST.2004.1338655

Repository homepage: https://researchrepository.rmit.edu.au

(C) 2004 IEEE

Downloaded On 2023/04/26 14:51:46 +1000

Please do not remove this page 


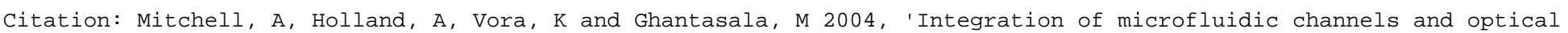

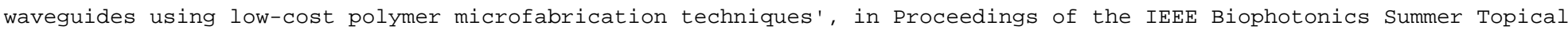
Conference 2004, San Diego, USA, 28-30 June 2004.

MA4.3

3:45 pm - 4:00 pm

\title{
Integration of Microfluidic Channels and Optical Waveguides using low-cost Polymer Microfabrication Techniques
}

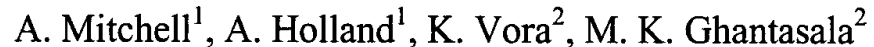 \\ ${ }^{1}$ School of Electrical and Computer Engineering, RMIT University, \\ GPO Box 2476V, Melbourne, VIC 3001, Australia \\ ${ }^{2}$ IRIS, Swinburne University of Technology, Melbourne, VIC 3122, Australia
}

\begin{abstract}
Recent progress on the realization of a silicon integrated biophotonic chip using plasma etching and laser ablation is presented. The chip utilizes films of SU-8 and UV-15 polymer material. An intersecting optical waveguide and microfluidic channel exhibiting good optical transmission across the channel is demonstrated.
\end{abstract}

\section{INTRODUCTION}

Recently, there has been great interest in the application of microtechnology to the realization of highly integrated biochips [1]. Devices of this type offer the potential for the rapid and flexible processing and analysis of very small sample volumes. These systems also benefit from the parallelisation that microfabrication and photolithography can offer. Further, if such biochips can be monolithically integrated with CMOS electronics, access to sophisticated signal processing and control can be made available, without the need for expensive packaging and data interconnects.

A popular application for biochip technology is the implementation of micro capillary electrophoresis systems [2]. Such systems can be used for the analysis of DNA. The use of microtechnologies enable the realization of systems that are a fraction of the size and cost of traditional implementations also greatly reducing the time required for processing samples. Such chips may thus be used for near instant diagnostics

The low cost, high-speed capabilities of biochips also make them attractive for more basic biological 'discovery' research. The capability of performing a multitude of experiments simultaneously on a single chip can render previously intractable experiments practical. An example of such an investigation that is of particular interest to us is the investigation of the role electromagnetic radiation plays in bioactivity of proteins and similar macromolecules [3].

The basic building block of a biochip is a micro-fluidic capillary [1] that can be used to channel a fluidic sample through the chip. For many biological applications, including those mentioned above $[2,3]$, photonic interrogation and/or activation of the sample is required. It is thus reasoned that two important structures for biochips are microfluidic channels and optical waveguides.

Microfluidic channels are often realized in bulk glass or polymer substrates [1]. Unfortunately, the dense integration of sophisticated electronics with such systems will require expensive packaging. Alternately, systems have been realized directly using CMOS silicon technology [4]. However, since silicon is highly absorbing at visible and ultraviolet optical wavelengths, photonic delivery must be conducted from the surface requiring complex photonic packaging or extemal bulk optics (e.g. a microscope). It would be advantageous to maintain a low cost monolithic biochip with minimal electronic and photonic packaging without compromising dense electronic integration and the range of optical wavelengths.
This investigation presents an approach to realizing integrated optical waveguides and microfluidic channels directly on silicon substrates. Optical waveguides realized using simple plasma etching techniques and are suspended between relatively thick polymer cladding layers. The microfluidic channels are formed by excimer laser ablation of trenches through the multiplayer wavegide structure. In this way the optical waveguides are effectively isolated from the silicon substrate, but directly interfaced to the microfluidic channel. Efficient optical transmission at visible wavelengths across a $50 \mu \mathrm{m}$ channel is demonstrated.

\section{BACKGROUND}

The optical materials selected for this investigation were UV-15 and SU-8. due to their ease of processing and low optical absorption to ultra-violet wavelengths $(<400 \mathrm{~nm})$. SU-8 has a high refractive index ( 1.6@633nm) compared to ( 1.45@633nm) for UV-15. This high index contrast enabled very small-scale waveguides to be realized. Multimode waveguides were deliberately targeted to ensure efficient transmission across the fluidic channel.

\section{WAVEGUIDE FABRICATION}

The waveguide fabrication procedure used in this investigation was similar to that reported in $[4,5]$. A 15 um film of UV-15 was applied to the Si substrate by spin coating at $2000 \mathrm{rpm}$. This film was cured by UV exposure and baking at $120 \mathrm{C}$ for $30 \mathrm{~min}$ and then sputter coated with $\mathrm{Al}$. The waveguide pattem was then etched into the $\mathrm{Al}$ and the sample was plasma etched in an $\mathrm{O}_{2}$ environment at $200 \mathrm{~W}$ for $20 \mathrm{~min}$ to form a $0.8 \mathrm{um}$ trench. An AFM image of such a trench is presented in Figure 1. A roughness of $<5 \mathrm{~nm}$ was measured.

The waveguide core was formed by spin coating a $1.5 \mu \mathrm{m}$ of SU-8 and curing by flood UV exposure and baking. A $20 \mu \mathrm{m}$ thick top cladding of UV-15 was then added to complete the waveguide structure. Figure 2 presents an AFM image of a polished end face. The differing polish quality of each of the polymer films provides contrast. The rib waveguide structure is evident.

\section{FLUIDIC CHANNEL FABRICATION}

Although the use of plasma etching to form microfluidic channels has been reported [6], excimer laser ablation was selected for this investigation. A $35 \mu \mathrm{m}$ deep, $50 \mu \mathrm{m}$ wide, $30 \mathrm{~mm}$ long fluidic channel was formed by dragging a rectangular exposure regiong. Two reservoir regions were also fabricated in this manner. Figure 3 presents a plan view of the intersecting microfluidic channel and polymer waveguides. The waveguides, (which are $20 \mathrm{~mm}$ beneath the surface) are in focus. 


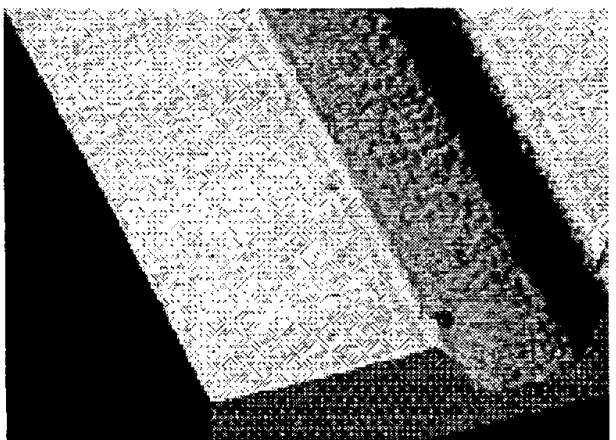

Fig. 1. Atomic fors micrograph of plasma etched waveguide trench

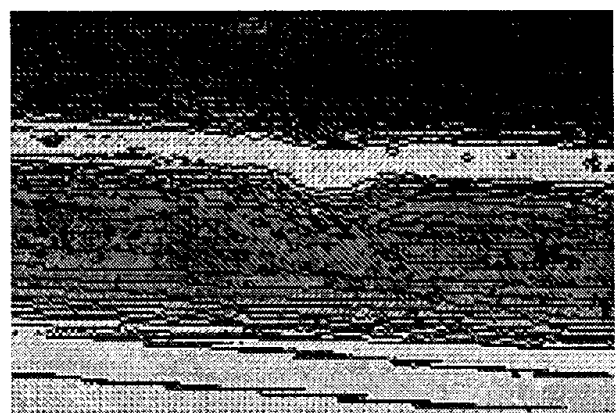

Fig. 2. Atomic force micrograph of polished waveguide cross-section

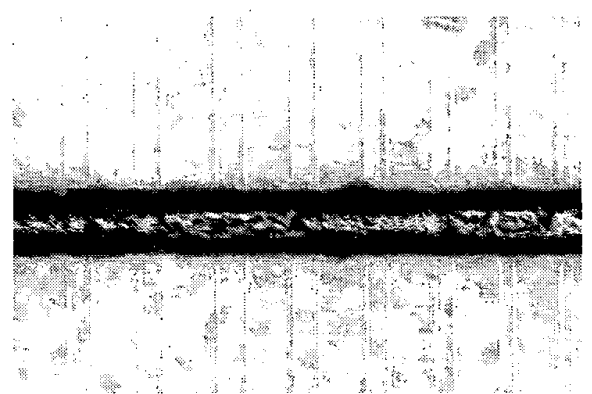

Fig. 3. Photograph of polymer optical waveguides with intersecting laser ablated micro-fluidic trench.
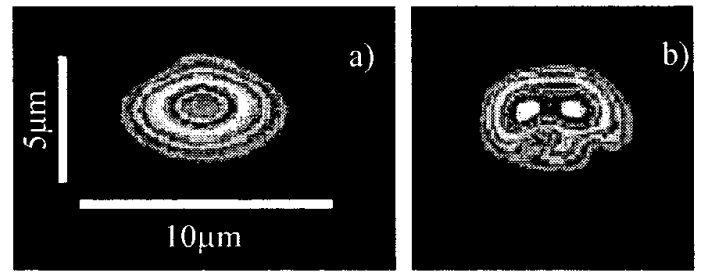

Fig. 4. Optical mode profiles: a) Mode profile of fundamental mode of optical waveguide b) optical mode profile after transmission across the micro-fluidic trench.

\section{WAVEguide CHARACTERIZATION}

Figure 5 presents CCD images of the mode profile measured at the output facet of the optical waveguide. Figure 5 a) presents the mode profile measured before introducing the trench. A tightly bound mode is evident. Figure $5 \mathrm{~b}$ ) presents the mode profile after introducing the trench. The roughness of the channel sidewall has resulted in excitation of a higher order waveguide mode. Efficient transmission of power is still evident, even across a $50 \mu \mathrm{m}$ air gap.

\section{CONCLUSIONS}

An intersection polymer optical waveguide and microfluidic channel has been demonstrated with efficient transmission of optical power. Sealing of the microfluidic channel and introduction of a fluidic sample has been attempted. Results of the photonic interrogation of fluidic samples will be presented at the workshop.

\section{ACKNOWLEDGMENT}

We acknowledge the Australian Photonics Cooperative Research Center for their contribution to this project and Shane Huntington, Department of Physics, The University of Melbourne for his exceptional atomic force microscopy.

\section{REFERENCES}

[1] C. Ahn, Choi Jin-Woo, G. Beaucage, J. Nevin, Lee Jeong-Bong, A.Puntambekar and J. Lee, "Disposable smart lab on a chip for point-of-care clinical diagnostics" Proc. of the IEEE, vol. 92, pp 154-173, Jan (2004)

[2] J. Webster, M. Burns, D. Burke, C. Mastrangelo, "Electrophoresis system with integrated on-chip fluorescence detection" Thirteenth Annual International Conference on Micro Electro Mechanical Systems MEMS 2000, pp. 306 - 310, Jan (2000)

[3] Cosic, I. "The Resonant Recognition Model of Macromolecular Bioactivity - Theory and Applications", Bikhauser Verlag (1997).

[4] H. Chena, T. Chena, S. Huanga, J. Gonga, J. Li, W. Chena, T. Hseub, I. Hsuc, "A novel micro-well array chip for liquid phase biomaterial processing and detection" Sensors and Actuators A, vol. 108, pp. 193200 (2003)

[5] A. Holland, A. Mitchell, H. Mendis and S. Huntington "A study of the plasma etching of thermoset polymers for waveguides", 3rd Internat. IEEE Conf. on Polymers and Adhesives In Microelectronics and Photonics Polytronics 2003, Montreux, Switzerland Oct, (2003).

[6] C. V. Cher, A. S. Holland, G. Rosengarten "Optimization techniques for plasma etching of thermoset polymer for microfluidic channels" Proceedings of the SPIE, vol. 5275, pp. 123-132, Dec (2003). 